\title{
Interactive Graphical Model Building using Telepresence and Virtual Reality *
}

\author{
Christopher Cooke \\ Massachusetts Institute of Technology \\ Cambridge, MA
}

\author{
Sharon Stansfield \\ Sandia National Laboratories \\ Albuquerque, NM
}

\section{Abstract}

This paper presents a prototype system developed at Sandia National Laboratories to create and verify computer-generated graphical models of remote physical environments. The goal of the system is to create an interface between an operator and a computer vision system so that graphical models can be created interactively. Virtual reality and telepresence are used to allow interaction between the operator, computer, and remote environment. A stereo view of the remote environment is produced by two CCD cameras. The cameras are mounted on a three degree-offreedom platform which is slaved to a mechanicallytracked, stereoscopic viewing device. This gives the operator a sense of immersion in the physical environment. The stereo video is enhanced by overlaying the graphical model onto it. Overlay of the graphical model onto the stereo video allows visual verification of graphical models. Creation of a graphical model is accomplished by allowing the operator to assist the inputer in modeling. The operator controls a 3-D cuisor to mark objects to be modeled. The computer then automatically extracts positional and geometric information about the object and creates the graphical hodel.

\section{Introduction}

Graphical models convey geometric information. Such models are usually created using tools such as computer-aided design (CAD) packages. However, building graphical models with a CAD system requires that the operator have geometric information for the objects to be modeled. This paper will discuss a prototype system for building graphical models that

- This work was performed at Sandia National Laboratories and was supported by the U.S. Department of Energy under Contract ???????

$$
D E-A C O H-94 A<85000
$$

does not require the operator to have a priori information about the objects in order to model them.

At Sandia National Laboratories, telerobotic applications, such as the retrieval of hazardous radioactive waste from underground storage tanks [4], create a need for building graphical models without a priori information about the remote site. These tanks are filled with obstacles such as cooling pipes, risers, and pumps. Since these sites are remote, and extremely dangerous, supervisory control of the robot by an operator is needed. However, the robot must generate its own motion plans. Without an accurate model, motion plans can be incorrect. Unfortunately, there is little available information about the geometry of the interior of these tanks; therefore an interactive graphical modeling system is proposed to allow modeling of the site from real-time sensor data.

The goal of this work is to provide such an intuitive interface between an operator, a vision system, and an interactive graphical modeler by using virtual reality and telepresence. The main component of the virtual reality system is a stereoscopic viewing device. To immerse the operator in a remote environment, the viewer displays live video from cameras located at the site. By slaving the motion of the cameras to the motion of the viewer - and hence the motion of the operator - direct natural control of the system is given to the operator. Since graphical information is also needed, motion-tracked graphical overlay capabilities are also provided. Other components of the virtual reality system are sound feedback, voice input, and motion tracking. Interfacing all these components to a modeling system creates an intuitive, easy to use interface. Motion tracking of the operator/cameras identifies scene position. $\Lambda 3-D$ pointer is used to specify objects in the live video. The opcrator guides the system by issuing voice commands. For example, when the operator says "cylinder," the system understands that the object to be modeled is a cylinder. Audio feedhack is provided to assist the op- 
erator in the modeling process. With guidance from the operator, the computer vision system is able to extract the necessary geometric information from the video and to place a representation of a previously unknown object into the graphical model.

Some work related to aspects of this project has been done: Cooper, et al. [5] use laser and video cameras to interactively create wireframe models of objects. Operator input is mouse and keyboard. Graphical overlays are possible. Stereo views and positioning of the camera are not addressed in the paper. Bon et al. [2] use stereo cameras to produce a stereo display on two flatscreen monitors. Wireframe models are interactively created. A joystick is used to mark points on the object in both views. The joystick is also used to control the view. Oxenberg et al. [8] use multiple cameras and a wireframe overlay to match remote vidco to a known model by interactively moving the graphical objects. Milgram et al. [7] use shutter glasses to display video and graphics. The purpose is to sludy human fusion of video and graphics. No models are created.

This work is unique in that it provides a stereo view of both the video and the graphics, with motion slaved to the motion of the user's head, thus immersing the user in the video/graphical model. It also provides interaction in the form of voice commands and voice recognition, and utilizes texture mapping and shaded graphics to produce a more realistic model.

\section{Prototype System}

\subsection{Software}

Modeling Software: SILMA's CimStation software is primarily a robotics simulation package, which can be extended and customized to provide added functionality. Extensions can be written in SIL, CimStation's native programming language) or linked in with code written in $\mathrm{C}$ or Fortran. This feature allows users of CimStation to customize their working environment. Hardware devices are interfaced to CimStation by linking in $\mathrm{C}$ code drivers.

CimStation was chosen for this work because of its extensibility and high-level graphics functions. Using SIL, graphical objects (eg. block, cylinder, sphere) can be created and positioned during execution. Preexisting models can be created and loaded using a menu-based interface.

Vision Software: 'The computer vision software used was specifically developed for this prototype system. It uses information input by the operator, such as rough object position and object identity, to guide computer vision processing. Lidge elements, or edgels, are extracted using an algorithm developed by Cammy [3]. The edgel information is used for stereo matching and triangulation to extract geometric and positional information about the object.

\subsection{Hardware}

The hardware used for this work consists of the following: A Silicon Graphics, Inc. Crimson/Reality Engine, a Silicon Graphics, Inc. Indigo sound server, a Fakespace Labs Boom 2C stereoscopic viewer, a Fakespace Labs Molly camera platform, a BG Systems Flybox, an RGB Spectrum frame grabber, a Dragon Systems voice recognition system, and an ultrasonic sensor.

The Boom is a six degree of freedom, stereoscopic viewer. It is mechanically tracked, producing both low latency and high accuracy. The resolution of the Boom, which is CRT based, is $1280 \times 1024$ pixels per channel. Molly is a three-degree-of-freedom platform with two monochrome CCD cameras mounted on it. Molly's motion is slaved to the yaw, pitch and roll of the Boom. Slaving the camera platform to the viewer gives the operator a sense of immersion in the remote environment. The stereo images also provide depth perception to the operator. Figure 1 shows the Boom/Molly system. The Flybox provides a 3-D joystick which is used to control the graphical pointer, allowing the operator to mark points in the live video. The ultrasonic sensor is mounted on the Molly platform. It has a range of 6 inches to 60 feet and is used to aid the operator in placing the graphical pointer in the 3-D image space. The voice recognition system is used for interpreting voice commands from the operator. The frame grabber is used by the computer vision system to capture digitized video images and to provide graphical overlay capabilities.

A system diagram is shown is Figure 2.

\subsection{Initialization of Video and Graph- ics}

First, Molly's two CCD video cameras nust be adjusted so that the parameters of both cameras match as closely as possible. These parameters include focal length, brightness, and contrast. Setting up the cameras in this manner allows the operator to fuse the two images into a $3-D$ view. In addition, the camera separation cannot be too large. Camera separation greater than $10 \mathrm{~cm}$ makes it difficult for the operator to fuse the images.

Registration of the stereo cameras with the stereo graphics is also required so that the graphics and the 
video match. Registration is accomplished by matching the graphical viewing parameters to the parameters derived by calibrating the two CCD cameras. $\Lambda$ least squared error technique is used to create a $4 \times 3$ camera matrix [1]. Once a matrix for each camera is calculated, the camera parameters can be extracted [6]. The camera matrix also provides a mapping from world points to image points. Using the parameters derived from the camera matrices, the stereo graphics can be registered with stereo video.

\subsection{Graphical Pointer}

A graphical pointer is used to mark objects in the video. Calibration, as discussed above, is used to ensure that the graphical and world positions coincide. The pointer is initially positioned using the ultrasonic sensor. The object in the center of the field of view is ranged, and the graphical pointer is placed at the depth of the object. This gives the operator an initial position measurement for the object. The operator then uses the joystick to control the position of the pointer, allowing him to mark points on the object in 3D space.

\section{Operator Interaction}

Verbal commands and/or Flybox button presses control the state of the modeling system. The computer informs the operator of the state of the system via audio feedback. Audio is also used to prompt the operator for input.

The system operates in two modes: verification and modeling. In the verification mode, Molly and the graphics are slaved to the Boom, with the graphics overlayed onto the live video from the cameras mounted on Molly. As the operator examines the remote environment, visual verification of the graphical models is possible. The graphical models may be displayed in wireframe or solid model form.

In the modeling mode, the operator freezes the graphics and camera motion using a voice command. By using the joystick to control a graphical pointer, the operator can mark points on an ouject. After marking the object the operator must identify the object (eg. box or cylinder) by issuing the appropriate verbal command. The computer vision system uses this information to extract positional and geometric information about the object, which is then placed in the graphical model of the environment.

\section{Computer Vision Processing}

The computer vision system is composed of two servers: the image server and the object server. 'The image server is responsible for grabbing the video images and performing edge detection. The object server then takes the images from the image server and extracts the dimension and pose (position and orientation) of the objects.

The image server is informed when modeling mode is entered. It then grabs a frame of video from each of the video cameras and performs edge detection [3] on those images. This provides a $3 \times 3$ direction derivative for each pixel. The edgel value and direction value are stored. A $3 \times 3$ direction derivative is used because the high-level image processing (location and identification) is being performed by the operator.

In the modeling mode, the user identifies the object as a cylinder or a box and marks four points which bound the object. This information is passed to the object server, along with the current orientation of the camera platform. The object server translates the four world points into eight image points (four for each image). These image points bound the edgels of interest.

If the object is identified as a cylinder, an edge is located that more precisely bounds the object. This edge is located by searching within the bounded area of the image for an edge that is closed. To find such an edge, an edge following routine was written as outlined by Schalkoff in [9]. The direction associated with each edgel of interest is stored in an array. This array is then rocessed to find possible corners. A possible corner is detected whenever there is a direction change of more than twenty degrees with respect to the edgel next to it. The four corners are determined by comparing the distance of all possible corners with each image point provided by the user.

The four corners in both images are then triangulated to give four world points. An assumption is made that the radius of the cylinder is smaller than its height. This assumption is not necessary if a constraint is placed on the user regarding the order in which the object is marked. With the four points and the above assumption, the dimensions and pose of the cylinder may be extracted. This method does not work well if the user is looking at the top or bottom of the cylinder. We assume that the user is able to correctly place the camera to model the object, and so this is not a problem.

If the object is identified as a box, all bounded edgels in the left image are extracted. The world points provided are then used to approximate the

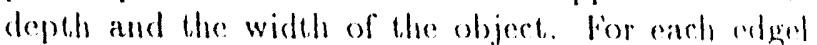


extracted, an epipolar line of pixels is calculated, in the right image, which would fall within that volume. Stereo malching in the form of auto correlation [9] $(10 \times 10)$ is then performed on the edgels and the line of epipolar pixels. The best match is then compared with a threshold to iviermine if the match is valid. This provides an array of world points that are associated with that object. These points are then bounded and model dimension and position are sent back to the graphics program. The bound is determined by the maximum and minimum in $x, y$, and $z$. The matched pixels in the left image also provide a bound on the image for texture mapping. By sorting the array for maximum and minimum image space $(u, v)$ values, a texture may be created and added to the face of the object. This texture is obtained from the digitized image of the object. Complex objects within the remote environment can then be stored within the graphical model as bounding boxes with a digitized image of the actuai object texture-mapped onto them. This provides added information and realism to the graphical environment.

\section{Results and Discussion}

The prototype system described here demonstrates that interactive graphical modeling of remote sites is viable. By interacting with both the live video of the site and the incomplete graphical model, operators can extend the graphical model to contain objects which were not previously known to exist. By using virtual reality and telepresence, an intuitive, easy-touse system was developed which requires little operator training to use. The prototype system was tested on several naive operators over the course of its development, and most of them were able to interactively create graphical models with only minor explanation and guidance.

The vision system is able to successfully extract and model cylinders and blocks. The block model may be used as a bounding box for more complex objects. Figure 3 shows a typical experimental set-up. The optical table is 52 inches by 43 inches. The cameras are approximately 25 inches from the table, or 25 to 75 inches from the objects in depth. 'The objects were 3 inches to 12 inches in height. Positions and dimensions of objects were measured and then compared against the values extracted by the vision system. In these preliminary experiments, objects were modeled with an accuracy within 0.25 inches of their actual dimensions. Depth placement gave the largest error due to the small camera separation, but was within 1.5 inches of actual depth. This accuracy is more than adequate for positioning robot manipulators and tools.

The current system is limited to camera motion about the $\mathrm{Z}$ axis (pan) only. 'This is due to positioning inaccuracies in the Molly camera platform. Future work with Fakespace Labs to increase the positioning accuracy of the Molly will alleviate this problem. The initial system configuration also contained a magnetic tracker, mounted on the operator's wrist, rather than a joystick, to control the graphical pointer. This provided a more intuitive method for controlling the position of the tracker (via natural movement of the operator's arm.) However, again due to the positioning inaccuracy of the device, the magnetic tracker was replaced by the joystick.

Depth placement of the graphical pointer was difficult for some operators who were not able to fuse the two images into a $3 \mathrm{D}$ view. This problem was alleviated by adding the ultrasonic sensor which alltomatically provides an initial depth measurement for the object.

Future work will include camera motion in all three degrees of freedom (pan, tilt, and roll) and extension of the computer vision system to allow modeling of ubjects with hidden edges. A larger toolkit of primitives with which to build graphical models will also be developed.

\section{Acknowledgements}

The authors would like to thank Larry Ray, Colin Selleck, and Chris Wilson for use of, and help with, the camera calibration software used for this work. Their contribution was vital to the success of this project.

\section{References}

[1] Ballard, D. H. and Brown, C. M. Computer Vi. sion, Prentice-Hall, Englewood Cliffs, New Jesrey, 1982.

[2] Bon, B., Wilcox, B., Litwin, T. and Gennery, D., "Operator-Coached Machine Vision for Space Telerobotics," SPIE Conference on Cooperative Intelligent Robotics in Space, Vol. 1387, pp. 337342,1990 .

[3] Canny, J. F', "A Computational Approach to Lige Detection," IEEE Transactions on Pattern Analysts and Machine Intelligence, vol. PAMI-8

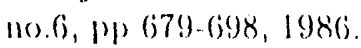




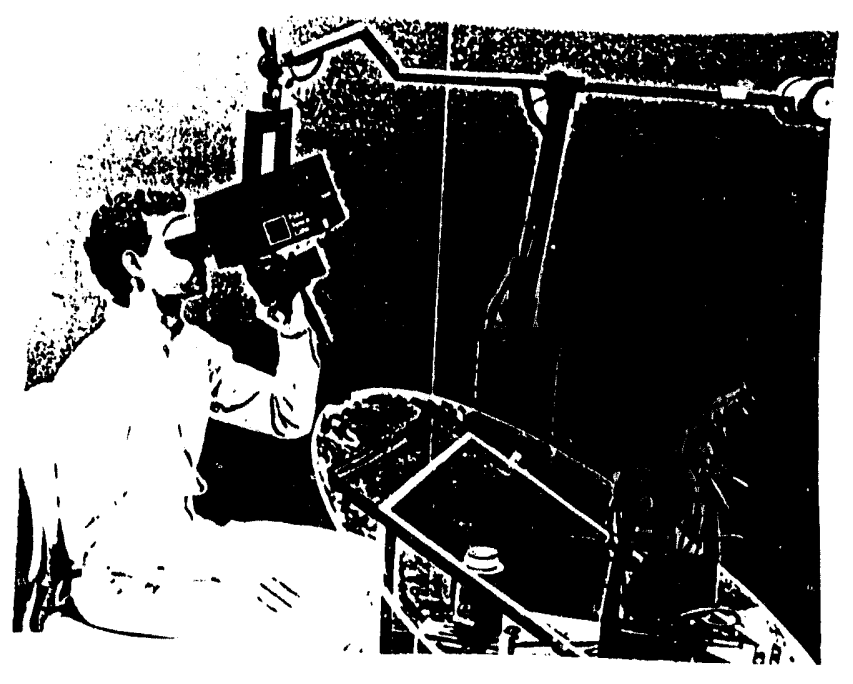

Figure 1: Boom/Molly System

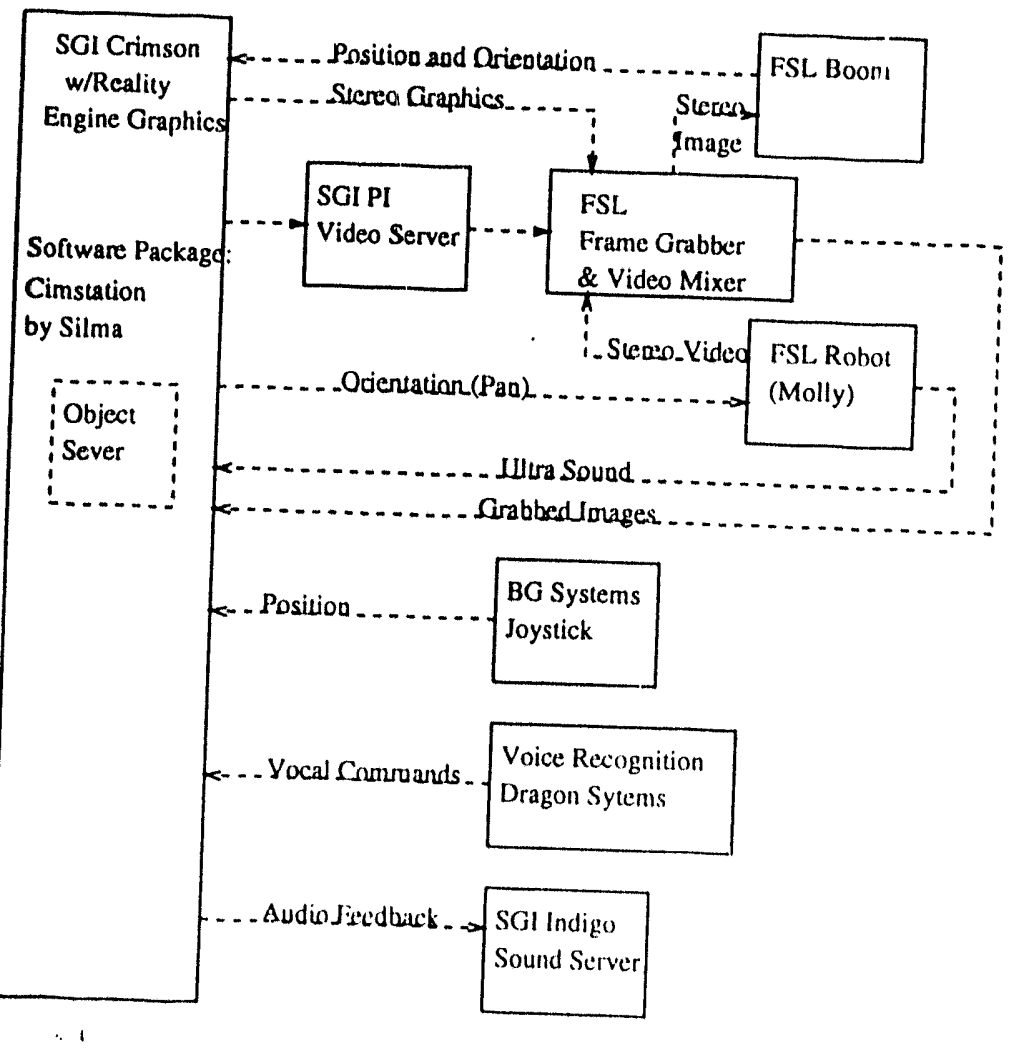

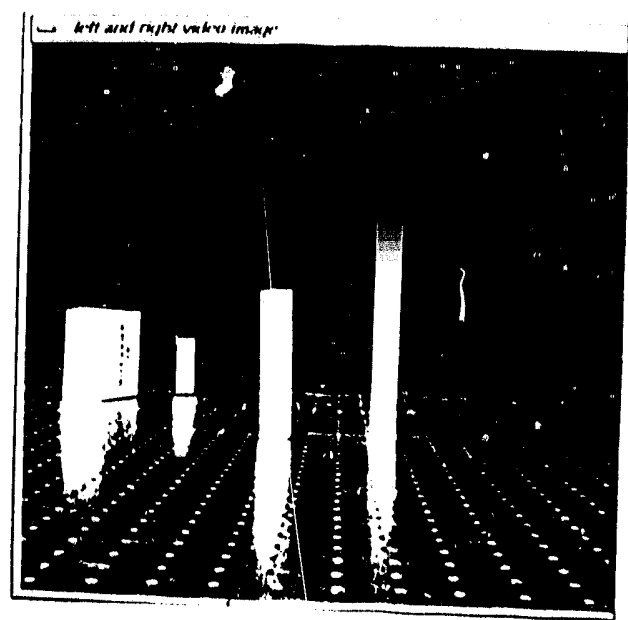

Figure 3: Experimental Set-up

[4] Christensen, B. K., Drotning, W. D., and Thunborg, S. "Model Based, Sensor Directed Remediation of Underground Storage Tanks", Journal of Robotic Systems, pp. 145-159, 1992

[5] Cooper, E., Vazquez S. and Goode P., "Interactive Scene Analysis Module: A Sensor Database Fusion System for Telerobotic Environments," SPIE Conference on Cooperative Intelligent Robotics in Space, Vol. 1621, pp. 334-345, 1991.

[6] Ganapathy, S. "Decomposition of Transformation Matrices for Robot Vision" IEEE Robotics pp.130-139, 1984.

[7] Milgram, P., Drascic, D. and Grodski, J., "A Virtual Stereographic Pointer for a Real Three Dimensional Video World," Proceedings of the IN. TERACT conference, pp. 695-700, 1990.

[8] Oxenberg, S., Landell, B. and Kan, E., "Geometric database maintenance using CCTV cameras and overlay graphics," SPIE Conference on Cooperative Intelligent Robotics in Space, Vol. 1006, pp. 115-123, 1988.

[9] Schalkoff, R. J. Digital Image Processing and Computer Vision. Wiley, New York, 1989.

\section{DISCLAIMER}

Figure 2: System Diagram
This report was prepared as an account of work sponsored by an agency of the United States Government. Neither the United States Government nor any agency thereof, nor any of their employees, makes any warranty, express or implied, or assumes any legal liability or responsibility for the accuracy, completeness, or usefulness of any information, apparatus, product, or process disclosed, or represents that its use would not infringe privately owned rights. Reference herein to any specific commercial product, process, or service by trade name, trademark, manufacturer, or otherwise does not necessarily constitute or imply its endorsement, recommendation, or favoring by the United States Government or any agency thereof. The views and opinions of authors expressed herein do not necessarily state or reflect those of the United States Government or any agency thereof. 

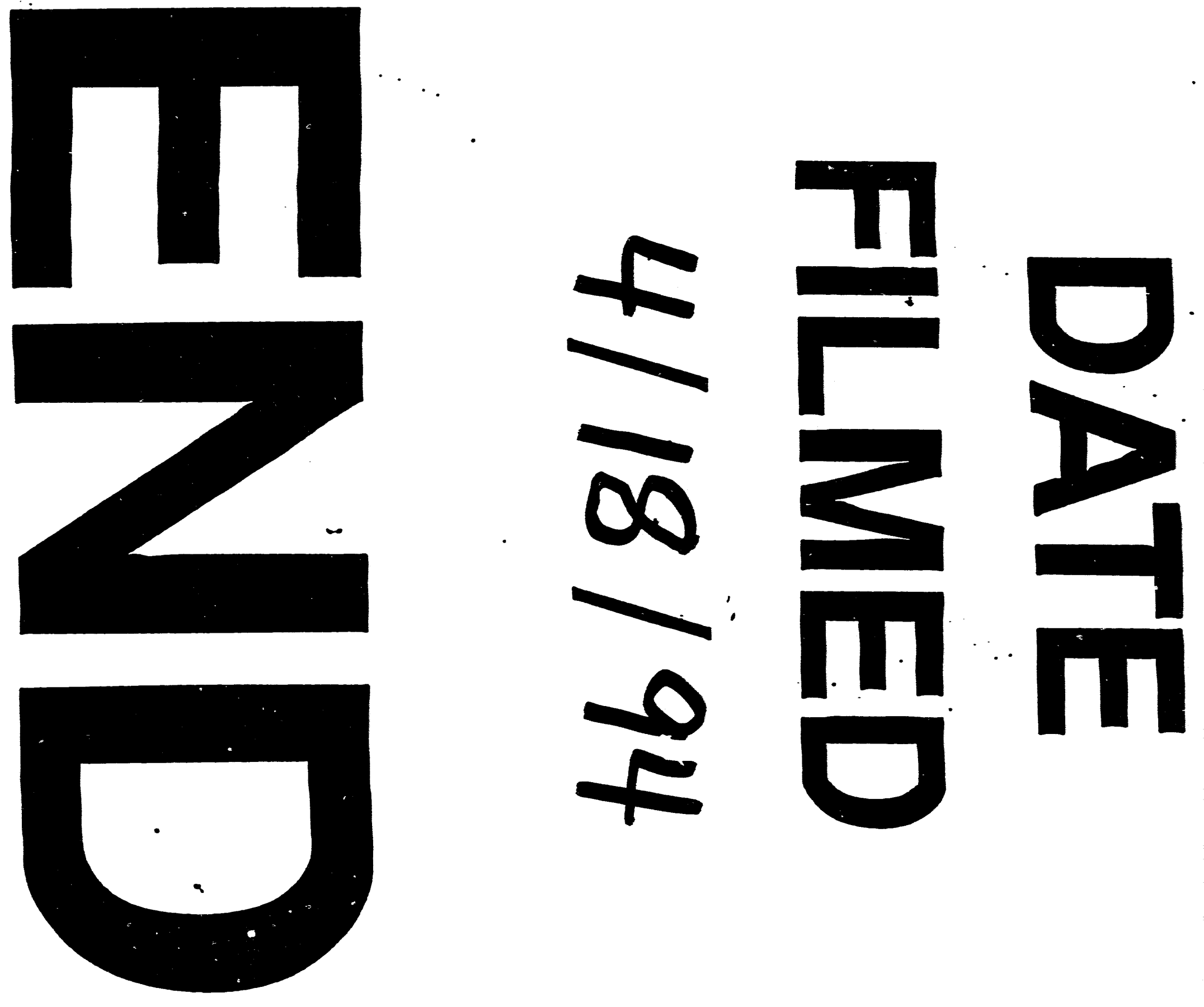
